\title{
Bupivacaine cardiotoxicity - hypercarbia is bad but hypocapnia may be worse
}

Derek Dillane MB BCH MMedSci FFARCSI, Brendan T. Finucane MB BCH BAO FRCPC FRCA

"When it appears a drug may cause serious side effects, physicians deserve careful presentation of the evidence."

So began an editorial concerning bupivacaine toxicity that appeared in this journal almost a quarter century ago. The editorial was written in response to a number of reports regarding cardiac arrest following regional anesthesia with bupivacaine. The cardiotoxic potential of bupivacaine became apparent when malignant ventricular arrhythmias and cardiac arrest, often refractory to prolonged resuscitation efforts, were reported in obstetric patients receiving epidural anesthesia. ${ }^{2}$ The subsequent introduction of multiple safety steps, including the use of an epinephrine test dose, fractionated dosing, and improved patient monitoring, led to an appreciable reduction, by a factor of up to 25 , in the overall incidence of systemic toxicity associated with epidural anesthesia. ${ }^{3}$ The contemporaneous search for a more innocuous, long lasting local anesthetic agent yielded, by way of chiral chemistry, the single enantiomers, levobupivacaine and ropivacaine. Each agent is prepared as a single levorotatory isomer with less potential for cardiac toxicity than its dextrorotatory congener in racemic bupivacaine. ${ }^{4}$

By and large, subsequent investigators have focused their attention on containment of the consequences of systemic toxicity. When unintentional intravascular injection does occur, the resultant maximum plasma concentration is dependent on the total dose injected, the speed and site of injection, and whether the injection is intravenous or intra-arterial. ${ }^{5}$ Arterial $\mathrm{CO}_{2}$ tension plays no small role. When elevated, it increases cerebral blood flow and further hastens delivery of local anesthetic to that target organ.

Indeed, local anesthetic pharmacology has long been associated with carbon dioxide. In the early 1960 's, attempts to prolong the duration of action of local anesthetic agents exploited the relationship with carbon dioxide, which was largely seen to be one of pharmacological potentiation. ${ }^{6}$ The ready diffusion of carbon dioxide through the nerve membrane leads to the formation of carbonic acid and a marked decrease in intraneural $\mathrm{pH}$. This favours the formation of the functional cationic form of the local anesthetic. Unable to readily permeate the nerve membrane, the active cation is effectively trapped, thus further enhancing the degree of conduction block.

A priori of this, hypercapnia / respiratory acidosis might be expected to exacerbate local anesthetic toxicity (enhance the negative inotropic and chronotropic effects of bupivacaine), an assumption supported by a number of experiments on animal models. ${ }^{7,8}$ Severe hypoxia, hypercapnia, and respiratory / metabolic acidosis are not unusual after local anesthetic-induced seizure activity. Accordingly, airway control, ventilation, and seizure suppression have long been a cornerstone of the treatment of local anesthetic toxicity. Rising toxicity or falling convulsant dose is evident with increasing carbon dioxide tension. Hyperventilation, according to de Jong, ${ }^{9}$ is the sensible first step in managing the initial cerebral aspect of local anesthetic toxicity, a recommendation that has subsequently been echoed by the American Heart Association's toxicology resuscitation guidelines. ${ }^{10}$

Conversely, in this issue of the Journal, Mochizuki et al. ${ }^{11}$ suggest that severe hypocapnic alkalosis may actually prolong resuscitation efforts following bupivacaine and levobupivacaine toxicity.

This well conducted investigation on an isolated rat heart model advocates for the maintenance of normocapnia when treating bradycardia following an unintentional intravascular injection of bupivacaine or levobupivacaine. It is suggested that ventilation to a

CAN J ANESTH 2008/55:12/pp. 807-812

From the Department of Anesthesiology and Pain Medicine, University of Alberta, Edmonton, Alberta, Canada.

Address correspondence to: Dr. Brendan T. Finucane, Department of Anesthesiology and Pain Medicine, University of Alberta,

Room CSB 8-120, 113 Street and 83 Avenue, Edmonton, Alberta T6G 2G3, Canada. Phone: 780-407-8861; Fax: 780-407-3200;

E-mail: bfinucan@ualberta.ca

Competing interests: None declared. 
$\mathrm{pCO}_{2} \leq 20 \mathrm{mmHg}$ with resultant severe respiratory alkalosis may actually prolong heart rate recovery during resuscitation efforts. This information sheds new light on the axiom endorsing hyperventilation during bupivacaine-induced cardiac toxicity. Interestingly, hypocapnic alkalosis did not prolong bradycardia at low concentrations of levobupivacaine $\left(1 \mu \mathrm{g} \cdot \mathrm{mL}^{-1}\right)$, but did prolong bradycardia at the same low concentration of bupivacaine. This may be further evidence in favour of the superior safety profile of the former agent. The exclusion of ropivacaine is a source for conjecture and as engaging as this study is, we must question our ongoing investigation of bupivacaine when a safer alternative was introduced into clinical practice over 12 years ago.

Despite the weight of incriminating evidence, bupivacaine is still the de facto standard among long-acting local anesthetic agents. ${ }^{12}$ In a survey of 135 US academic anesthesia departments, $55 \%$ of respondents had a preference for bupivacaine as a sole long-acting agent; $43 \%$ routinely use ropivacaine as their agent of choice; and levobupivacaine was selected by the remainder. ${ }^{13}$ Factors other than safety, such as onset time, perceived quality, and duration of nerve blockade clearly influence choice of local anesthetic. However, is there any evidence for our dyed in the wool doctrine regarding choice of long-acting local anesthetic agents?

Much controversy and popular opinion surrounds the true equipotency ratio among the three longacting agents. Results from a number of animal and clinical studies would suggest a rank order of potency of ropivacaine < levobupivacaine < bupivacaine. ${ }^{4}$ This might suggest that, due to its lower potency, any theoretical cardioprotective benefit derived from ropivacaine would be negated by the clinical need for higher doses. The difference in potency does not appear to be clinically relevant for surgical blocks (both peripheral and epidural) when the newer agents are used at concentrations of $0.5-0.75 \%$, the clinical profile of the nerve block being similar to that obtained with racemic bupivacaine. However, the lower potency of ropivacaine becomes relevant when used for postoperative analgesia with both epidural and continuous peripheral nerve blockade. For this application, $0.2 \%$ ropivacaine appears to be as effective as $0.125-0.15 \%$ levobupivacaine, which, in turn, is identical to racemic bupivacaine. ${ }^{4}$ In light of this evidence, our fidelity to bupivacaine appears to be unfounded, particularly with regard to epidural and peripheral nerve anesthesia. In the interests of safety, the high concentrations required to achieve surgical anesthesia and the high volumes required for postoperative analgesia would be better served by one of the single enantiomer agents.
Despite the enhanced safety profile of ropivacaine and levobupivacaine, they remain potentially lifethreatening, and, as such, recent advances in containment strategies are not redundant. Lipid resuscitation may be the very essence of this mode of therapy. The mechanism of action of lipid emulsion has not been fully elucidated, but it may act as a circulating lipid sink extracting lipophilic local anesthetic from plasma or tissues. ${ }^{14}$ An alternative proposed mechanism is the reversal of local anesthetic inhibition of myocardial fatty acid oxidation, thereby restoring myocardial adenosine triphosphate supply. ${ }^{15}$ Weinberg et al. ${ }^{16}$ conducted the original research involving the successful resuscitation of rats in which cardiovascular collapse was induced with intravenous bupivacaine. There has been a recent proliferation of case reports regarding the successful use of lipid emulsion in cardiovascular collapse associated with ropivacaine and levobupivacaine as well as bupivacaine. In addition to addressing the issue of efficacy, we have been given a valuable insight into many cases of cardiac toxicity that may otherwise have gone unreported. These reports also serve as a timely reminder that frequent negative aspiration, slow incremental injection, and the addition of an epinephrine marker do not preclude intravascular injection. ${ }^{17-21}$

Staying in the domain of the experimental, a number of recent in vipo and in vitro studies have shown some value in using an insulin-glucose-potassium infusion when treating both racemic and levorotatory bupivacaine toxicity. Postulated mechanisms of action include effects on $\mathrm{Na}+$ and $\mathrm{K}+$ channel dynamics, in addition to calcium homeostasis, which may improve myocardial conduction and contractility. ${ }^{22}$

The introduction and popular acceptance of ultrasound-guided regional anesthesia may be as consequential for averting local anesthetic systemic toxicity as the pharmacological advances of previous decades. For the first time in the century-old practice of regional anesthesia, it is possible to visualize the target neural structure adjacent to potential vascular hazards, thus permitting the more accurate deposition of smaller volumes of local anesthetic. In addition, slow injection allows for direct visualization of the spread of local anesthetic solution, which ensures increased confidence in exact localization. In every reported case of the successful use of lipid emulsion therapy to date, ultrasound guidance has been conspicuous by its absence. Due to the concern regarding needle movement, nerve stimulation techniques alone may encourage rapid injection of local anesthetic. The use of ultrasound does not negate the need for the more conventional safety mechanisms; however, when used in combination, they approach an entirely new scientific proposition. 
Immediate intervention at the earliest sign of toxicity is of prime importance and improves the chances of successful treatment. The supportive measures that are warranted include Advanced Life Support Guidelines and further specific measures directed at local anesthetic toxicity. On the basis of available evidence, it would appear apposite to instigate lipid emulsion therapy at the earliest signs of toxicity. All patients subject to a regional anesthesia procedure must undergo electrocardiography, pulse oximetry, and blood pressure monitoring. Bearing in mind that hypoxia, hypercarbia, and acidosis each worsen prognosis, oxygen therapy is obligatory and over sedation must be avoided. In the event of toxicity, airway management or seizure control must not be delayed.

The evidence against bupivacaine, as summoned, has been accumulating for the past 30 years. In addition to the popular call for a place for lipid emulsion therapy in every regional anesthesia cart, is it conceivable that we might create the necessary space by removing bupivacaine? Prevention rather than containment is now de rigueur in many medical specialties. Rather than concentrating our investigative efforts on a superannuated local anesthetic, perhaps we should finally embrace the safer alternatives.

\section{Cardiotoxicité de la} bupivacaïne : l'hypercapnie est mauvaise, mais l'hypocapnie peut être pire

\footnotetext{
"Lorsqu'il y a lieu de croire qu'un médicament possède des effets secondaires graves, il est essentiel de mettre au courant les médecins eux-mêmes. »l

Ainsi s'ouvrait un éditorial qui portait sur la toxicité de la bupivacaïne paru dans cette revue il y a près d'un quart de siècle. L'éditorial avait été rédigé en réponse à plusieurs comptes-rendus concernant des arrêts cardiaques survenus à la suite d'une anesthésie
}

régionale réalisée avec de la bupivacaïne. Le potentiel cardiotoxique de la bupivacaine est devenu apparent lorsque des cas d'arythmie ventriculaire maligne et d'arrêt cardiaque, souvent réfractaires à des efforts soutenus de réanimation, ont été rapportés chez des parturientes recevant une anesthésie péridurale. ${ }^{2}$ L'introduction subséquente de multiples mesures de sécurité, notamment l'utilisation d'une dose de test avec épinéphrine, d'un dosage fractionné, ainsi qu'un meilleur monitorage des patientes, a permis une réduction appréciable - jusqu'à 25 fois - de l'incidence globale de toxicité systémique associée à l'anesthésie péridurale. ${ }^{3} \mathrm{La}$ recherche contemporaine d'un agent anesthésique plus inoffensif et à action plus longue a eu pour résultat, en se basant sur la chimie chirale, les énantiomères simples : la lévobupivacaïne et la ropivacaïne. Chaque agent est préparé comme un isomère lévogyre simple avec moins de potentiel de toxicité cardiaque que son pendant dextrogyre présent dans la bupivacaïne racémique. ${ }^{4}$

Par la suite, les chercheurs ont pour la plupart concentré leur attention sur le moyen de limiter les conséquences de la toxicité systémique. Lorsqu'une injection intravasculaire involontaire survient, la concentration plasmatique maximale qui en résulte dépend de la dose totale, du site de l'injection et de sa vitesse, et du fait que l'injection est intraveineuse ou intra-artérielle. ${ }^{5} \mathrm{La}$ tension de $\mathrm{CO}_{2}$ artériel joue un rôle capital. Lorsqu'elle est élevée dans l'organe cible, elle augmente le débit sanguin au cerveau et accélère encore plus l'apport d'anesthésique local.

La pharmacologie des anesthésiques locaux a longtemps été associée au dioxyde de carbone. Au début des années 60 , certaines méthodes ont tenté de prolonger la durée d'action des agents anesthésiques locaux et ont exploité cette relation avec le dioxyde de carbone, considéré par beaucoup comme une potentialisation pharmacologique. ${ }^{6} \mathrm{La}$ diffusion facile du dioxyde de carbone à travers la membrane nerveuse génère la formation d'acide carbonique ainsi qu'une diminution marquée du $\mathrm{pH}$ intraneural. Ceci favorise la formation de la forme fonctionnelle cationique de l'anesthésique local. En effet le cation actif, incapable d'infiltrer facilement la membrane nerveuse, est pris au piège, ce qui améliore encore plus le degré du bloc de conduction.

Cela étant, on pourrait penser que l'hypercapnie / acidose respiratoire exacerberait la toxicité de l'anesthésique local (c.-à-d. augmenterait les effets inotropiques et chronotropiques négatifs de la bupivacaïne), une hypothèse soutenue par plusieurs expériences menées sur des modèles animaux. ${ }^{78}$ L'hypoxie grave, l'hypercapnie et l'acidose respiratoire / métabolique 
ne sont pas rares après une crise induite par un anesthésique local. Par conséquent, le contrôle des voies aériennes, la ventilation et la suppression de la crise ont longtemps constitué la pierre angulaire du traitement de la toxicité de l'anesthésique local. Une toxicité accrue ou une dose de convulsivant qui chute sont deux des mécanismes mis en évidence par une tension croissante de dioxyde de carbone. Selon de Jong, ${ }^{9}$ l'hyperventilation est la première mesure raisonnable pour prendre en charge l'aspect cérébral initial de la toxicité de l'anesthésique local. Cette recommandation a par la suite été reprise par les directives de réanimation de toxicologie de l'American Heart Association. ${ }^{10}$

En revanche, dans ce numéro du Journal, Mochizuki $e t$ coll. ${ }^{1}$ suggèrent qu'une alcalose hypocapnique grave pourrait en fait prolonger les efforts de réanimation après une toxicité à la bupivacaïne ou la lévobupivacaïne.

Cette étude réalisée avec soin sur un modèle de cour de rat isolé prône le maintien de la normocapnie lors du traitement d'une bradycardie survenant à la suite d'une injection intravasculaire involontaire de bupivacaïne ou de lévobupivacaïne. Les auteurs suggèrent qu'une ventilation à une $\mathrm{pCO}_{2} \leq 20 \mathrm{mmHg}$ accompagnée de l'alcalose respiratoire grave qui en découle pourrait en fait prolonger le temps nécessaire au rétablissement de la fréquence cardiaque pendant les efforts de réanimation. Cette information donne une nouvelle perspective sur l'axiome appuyant l'hyperventilation comme moyen de surmonter une toxicité cardiaque induite par bupivacaïne. Fait intéressant, l'alcalose hypocapnique n'a pas prolongé la bradycardie lorsque des concentrations faibles de lévobupivacaïne $\left(1 \mu \mathrm{g} \cdot \mathrm{mL}^{-1}\right)$ étaient utilisées, mais a prolongé la bradycardie avec la même faible concentration de bupivacaïne. Il se peut que des données probantes supplémentaires soient nécessaires pour déterminer le profil d'innocuité supérieur de la lévobupivacaïne. L'exclusion de la ropivacaïne de cette étude donne lieu à des conjectures. Malgré le côté séduisant de cette étude, nous devrions tout de même remettre en question la pertinence de recherches continues sur la bupivacaïne lorsqu'une option plus sécuritaire a été introduite dans la pratique clinique il y a plus de 12 ans.

Malgré la quantité importante de données probantes compromettantes, la bupivacaïne demeure la norme de facto parmi les agents anesthésiques locaux à action prolongée. ${ }^{12}$ Dans un sondage réalisé auprès de 135 départements universitaires d'anesthésie aux États-Unis, $55 \%$ des répondants ont mentionné leur préférence pour la bupivacaïne en tant qu'agent à action prolongée unique ; $43 \%$ utilisent la ropivacaïne de façon routinière en tant que leur agent anesthésique préféré ; et le reste des répondants a admis préférer la lévobupivacaine..$^{13}$ Outre la sécurité, d'autres facteurs tels que le délai d'installation, la qualité perçue, et la durée du bloc nerveux influencent clairement le choix de l'anesthésique local. Cependant, existe-t-il réellement des preuves sur lesquelles se fonde notre doctrine profondément ancrée quant au choix d'agents anesthésiques locaux à action prolongée ?

La controverse et les opinions populaires sont foison autour du niveau réel d'équipotence entre ces trois agents à action prolongée. Les résultats de plusieurs études animales et cliniques suggèrent un ordre de puissance ropivacaïne < lévobupivacaïne < bupivacaïne. ${ }^{4} \mathrm{Ce}$ classement pourrait suggérer que, en raison de sa puissance plus faible, tout bienfait cardioprotecteur théorique provenant de la ropivacaïne serait annulé par l'exigence clinique de doses plus élevées. La différence de puissance ne semble pas avoir de pertinence clinique lorsqu'il s'agit de blocs chirurgicaux (périphériques et périduraux) lorsque les agents plus récents sont utilisés à des concentrations de 0,5 $0,75 \%$, étant donné que le profil clinique du bloc nerveux est semblable à celui obtenu avec de la bupivacaïne racémique. Néanmoins, la puissance plus faible de la ropivacaîne devient pertinente lorsque cet agent est utilisé en analgésie postopératoire dans le cadre de blocs nerveux périduraux et périphériques continus. Dans ce contexte, la ropivacaïne $0,2 \%$ semble être aussi efficace que la lévobupivacaïne $0,125-0,15 \%$, ou la bupivacaïne racémique aux mêmes concentrations. ${ }^{4}$ À la lumière de ces données, notre fidélité à la bupivacaïne semble sans fondement, particulièrement en ce qui concerne l'anesthésie péridurale et celle des nerfs périphériques. Par souci de sécurité, les concentrations élevées qui sont nécessaires pour obtenir une anesthésie chirurgicale et les volumes élevés nécessaires à l'analgésie postopératoire seraient mieux réalisés avec l'un des agents énantiomères uniques.

Malgré le meilleur profil d'innocuité de la ropivacaïne et de la lévobupivacaïne, ces deux agents demeurent tout de même potentiellement fatals. Dès lors, les progrès récents réalisés dans les stratégies de limitation des dégâts ne sont pas superflus. La réanimation lipidique pourrait bien constituer l'essence même de ce type de traitement. Le mécanisme d'action de l'émulsion lipidique n'a pas encore été complètement élucidé, mais elle pourrait agir comme un 'drain' lipidique circulant permettant l'extraction de l'anesthésique local lipophile du plasma et des tissus. ${ }^{14}$ Un autre mécanisme proposé est l'inversion de l'inhibition, par l'anesthésique local, de l'oxydation des acides gras myocardiques, restaurant ainsi l'approvi- 
sionnement d'adénosine triphosphate au myocarde. ${ }^{15}$ Les recherches originales sur la réanimation réussie de rats chez lesquels une défaillance cardiovasculaire avait été provoquée avec de la bupivacaine intraveineuse ont été réalisées par Weinberg et coll. ${ }^{16}$ Récemment, nous avons vu une prolifération des présentations de cas portant sur l'utilisation réussie d'émulsion lipidique dans les cas de défaillance cardiovasculaire associée à la ropivacaïne ou à la lévobupivacaïne, ainsi qu'à la bupivacaine. Outre le fait que ces études traitaient des questions d'efficacité de ces agents, elles nous ont également permis d'observer plusieurs cas de toxicité cardiaque qui auraient pu ne pas être rapportés autrement. Ces présentations sont également un rappel opportun que l'aspiration négative fréquente, l'injection lente de doses fractionnées et l'ajout d'un marqueur d'épinéphrine n'excluent pas l'injection intravasculaire..$^{17-21}$

Toujours dans le domaine expérimental, nombre d'études récentes in vivo et in vitro ont montré que l'utilisation d'une perfusion d'insuline-glucosepotassium pouvait s'avérer utile lors du traitement de la toxicité de la bupivacaine racémique et lévogyre. Les mécanismes d'action possibles comprennent les effets sur l'homéostasie calcique, mais aussi sur la dynamie des canaux $\mathrm{Na}+$ et $\mathrm{K}+$, ce qui pourrait améliorer la conduction et la contractilité myocardiques. ${ }^{22}$

L'introduction et l'acceptation grandissante de l'anesthésie régionale échoguidée pourrait avoir autant de conséquences sur l'évitement de la toxicité systémique de l'anesthésique local que les progrès pharmacologiques des décennies passées. Pour la première fois dans la pratique centenaire de l'anesthésie, il est possible de visualiser la structure neurale ciblée et d'éviter les structures vasculaires potentiellement à risque, permettant un placement plus précis de volumes moindres d'agents anesthésiques. De plus, une injection lente permet de visualiser directement la diffusion de la solution d'anesthésique local, ce qui augmente le niveau de certitude quant à la localisation exacte. À ce jour, dans chaque cas rapporté qui traite de l'utilisation réussie d'un traitement à base d'émulsion lipidique, l'échoguidage brille par son absence. En raison des inquiétudes concernant le mouvement de l'aiguille, les techniques de stimulation nerveuse sont les seules à justifier une injection rapide de l'anesthésique local. L'utilisation de l'échoguidage ne va pas à l'encontre du besoin que nous avons des mécanismes de sécurité traditionnels ; néanmoins, lorsque ces techniques sont combinées, elles créent une proposition scientifique tout à fait nouvelle.

Une intervention immédiate dès les premiers signes de toxicité est primordiale et améliore les chances de succès du traitement. Parmi les autres mesures de soutien justifiées, citons les Directives des soins spécialisés en réanimation cardio-respiratoire et d'autres mesures spécifiques qui se concentrent sur la toxicité de l'anesthésique local. Si l'on se fonde sur les données probantes disponibles, il semblerait pertinent de mettre en place un traitement avec émulsion lipidique dès les premiers signes de toxicité. Tous les patients se présentant pour une intervention avec une anesthésie régionale doivent subir les examens suivants : électrocardiographie, oxymétrie de pouls et monitorage de la pression artérielle. Sachant que l'hypoxie, l'hypercarbic et l'acidose ont toutes un effet négatif sur le pronostic, l'oxygénothérapie est de mise et une sédation excessive doit être évitée. $\mathrm{Si}$ une toxicité survient, la prise en charge des voies aériennes ou le contrôle de la crise doivent être réalisés dans les plus brefs délais.

Les données probantes allant contre la bupivacaïne, lorsque rassemblées, s'accumulent depuis une trentaine d'années. Outre l'exhortation populaire pour qu'il y ait une place pour le traitement avec émulsion lipidique dans chaque chariot d'anesthésie régionale, est-il concevable de faire cette place en éliminant la bupivacaïne? Prévenir, plutôt que limiter les dégâts, est désormais de rigueur dans de nombreuses spécialités médicales. Plutôt que de concentrer nos efforts de recherche sur un anesthésique local obsolète, nous devrions peut-être, en fin de compte, adopter ses alternatives plus sécuritaires.

\section{References}

1 Writer WD, Davies JM, Strunin L. Trial by media: the bupivacaine story. Can Anaesth Soc J 1984; 31: 1-4.

2 Albright GA. Cardiac arrest following regional anesthesia with etidocaine or bupivacaine. Anesthesiology 1979; 51: 285-7.

3 Mulroy MF. Systemic toxicity and cardiotoxicity from local anesthetics: incidence and preventive measures. Reg Anesth Pain Med 2002; 27: 556-61.

4 Casati A, Putzu M. Bupivacaine, levobupivacaine and ropivacaine: are they clinically different? Best Pract Res Clin Anaesthesiol 2005; 19: 247-68.

5 Covino BG, Vassalo $H G$. Pharmacokinetic aspects of local anesthetic agents. In: Covino BG, Vassalo HG (Eds). Local Anesthetics. Mechanisms of Action and Clinical Use. New York: Grune \& Stratton; 1976: 95-121.

6 Condouris $G A$, Shakalis $A$. Potentiation of the nervedepressant effect of local anaesthetics by carbon dioxide. Nature 1964; 204: 57-8.

7 Rosen MA, Thigpen JW, Shnider SM, Foutz SE, 
Levinson G, Koike M. Bupivacaine-induced cardiotoxicity in hypoxic and acidotic sheep. Anesth Analg 1985; 64: 1089-96.

8 Sage DJ, Feldman HS, Arthur R, et al. Influence of lidocaine and bupivacaine on isolated guinea pig atria in the presence of acidosis and hypoxia. Anesth Analg $1984 ; 63: 1-7$.

9 de Jong $R H$. Central nervous system effects. In: de Jong RH (Ed.). Local Anesthetics. St. Louis: Mosby; 1994: 288.

102005 American Heart Association Guidelines for cardiopulmonary resuscitation and emergency cardiovascular care, Part 10.2: Toxicology in emergency cardiovascular care. Circulation 2005; 112(suppl IV): IV-126-32.

11 Mochizuki T, Sato S. Hypocapnia prolongs bradycardia induced by bupivacaine or levobupivacaine in isolated rat hearts. Can J Anesth 2008; 55: 836-46.

12 Mather LE, Chang D. Cardiotoxicity with modern local anaesthetics: is there a safer choice? Drugs 2001; 61: $333-42$.

13 Corcoran W, Butterworth J, Weller RS, et al. Local anesthetic-induced cardiac toxicity: a survey of contemporary practice strategies among academic anesthesiology departments. Anesth Analg 2006; 103: 1322-6.

14 Weinberg $G$, Ripper $R$, Feinstein DL, Hoffman W. Lipid emulsion infusion rescues dogs from bupivacaineinduced cardiac toxicity. Reg Anesth Pain Med 2003; 28: 198-202.

15 Picard J, Meek T. Lipid emulsion to treat overdose of local anaesthetic: the gift of the glob. Anaesthesia 2006; 61: 107-9.

16 Weinberg $G L$, VadeBoncouer $T$, Ramaraju $G A$, Garcia-Amaro MF, Cwik MJ. Pretreatment or resuscitation with a lipid infusion shifts the doseresponse to bupivacaine-induced asystole in rats. Anesthesiology 1998; 88: 1071-5.

17 Rosenblatt MA, Abel M, Fischer GW, Itzkovich CJ, Eisenkraft JB. Successful use of a $20 \%$ lipid emulsion to resuscitate a patient after a presumed bupivacainerelated cardiac arrest. Anesthesiology 2006; 105 : 217-8.

18 McCutchen T, Gerancher JC. Early intralipid therapy may have prevented bupivacaine-associated cardiac arrest. Reg Anesth Pain Med 2008; 33: 178-80.

19 Warren JA, Thoma RB, Georgescu A, Shab SJ. Intravenous lipid infusion in the successful resuscitation of local anesthetic-induced cardiovascular collapse after supraclavicular brachial plexus block. Anesth Analg 2008; 106: 1578-80.

20 Litz RJ, Popp M, Stels SN, Koch T. Successful resuscitation of a patient with ropivacaine-induced asystole after axillary plexus block using lipid infusion. Anaesthesia 2006; 61: 800-1.

21 Foxall G, McCahon R, Lamb J, Hardman JG, Bedforth NM. Levobupivacaine-induced seizures and cardiovascular collapse treated with intralipid. Anaesthesia 2007; 62: 516-8.

22 Stehr SN, Pexa A, Hannack S, et al. Insulin effects on myocardial function and bioenergetics in L-bupivacaine toxicity in the isolated rat heart. Eur J Anaesthesiol $2007 ; 24: 340-6$. 